\title{
Filtering Techniques for Chaotic Signal Processing
}

\author{
Denis Butusov ${ }^{1, *} \mathbb{C}^{\circ}$, Timur Karimov ${ }^{2}{ }^{\oplus}$, Alexander Voznesenskiy ${ }^{3}$, Dmitry Kaplun ${ }^{3}{ }^{\oplus}$, \\ Valery Andreev ${ }^{2}$ and Valerii Ostrovskii ${ }^{2}$
}

1 Youth Research Institute, St. Petersburg Electrotechnical University “LETI”, St. Petersburg 197376, Russia

2 Department of Computer-Aided Design, St. Petersburg Electrotechnical University "LETI", St. Petersburg 197376, Russia; tikarimov@etu.ru (T.K.); vsandreev@etu.ru (V.A.); vyostrovskii@etu.ru (V.O.)

3 Department of Automation and Control Processes, St. Petersburg Electrotechnical University "LETI", St. Petersburg 197376, Russia; a-voznesensky@yandex.ru (A.V.); dikaplun@etu.ru (D.K.)

* Correspondence: dnbutusov@etu.ru; Tel.: +7-950-008-71-90

Received: 16 November 2018; Accepted: 17 December 2018; Published: 19 December 2018

check for updates

\begin{abstract}
The vulnerability of chaotic communication systems to noise in transmission channel is a serious obstacle for practical applications. Traditional signal processing techniques provide only limited possibilities for efficient filtering broadband chaotic signals. In this paper, we provide a comparative study of several denoising and filtering approaches: a recursive IIR filter, a median filter, a wavelet-based denoising method, a method based on empirical modes decomposition, and, finally, propose the new filtering algorithm based on the cascade of driven chaotic oscillators. Experimental results show that all the considered methods make it possible to increase the permissible signal-to-noise ratio to provide the possibility of message recognition, while the new proposed method showed the best performance and reliability.
\end{abstract}

Keywords: chaos; communication systems; chaotic synchronization; denoising; secure communications; digital signal processing; filtering; empirical modes decomposition

\section{Introduction}

Chaotic communication has number of advantages over traditional harmonic signal-based schemes, namely, better security and steganographic properties [1], low power spectrum density, robustness to multi-path fading and ease of wideband communication system implementation [2]. Chaotic communications are also of interest due to the wide bandwidth of chaotic signals making them useful in systems where narrowband channels are overloaded [3].

Today a number of practical implementations of chaotic communication systems are known [4]. At the early stage of investigations in the field of chaotic communication, several simple methods have been proposed, including chaotic masking, chaotic regime switching, the nonlinear mixing of the information into the chaotic carrier, etc. Later, it was found that these methods suffer from low robustness to noise in the transmission channel $[1,4,5]$. To overcome this drawback, a number of techniques have been introduced, which can be divided into two main classes: noise-stable communication schemes and conventional communication schemes with noise reduction; some hybrid types are reported as well. Noise-stable communication approaches include communication with use of generalized synchronization [3,5], the use of analytically-solvable chaotic systems [6], correlation detection with symbolic dynamic encoding [7], etc. Noise reduction techniques for chaotic signals cannot take advantage of the simple signal spectra of conventional multi-harmonic communication systems, forcing them to use linear FIR or IIR filters. Therefore, only relatively complex techniques are feasible for chaotic signal denoising. These approaches include noise reduction methods via a deterministic optimization technique [8], wavelet denoising [9], hidden Markov model approach [10], 
and the usage of Kalman filter for noisy state variables estimation [11]. Nevertheless, no generally accepted technique is known to date, and practical comparisons of these approaches are fragmentary and scarce.

In our work, we study two more filtration techniques which we believe not to have been reported yet in the context of chaotic communications: empiric mode decomposition denoising and cascading of driven chaotic oscillators. We compare these techniques with wavelet denoising, median filtering and IIR filtering using the example of a chaotic communicational system model with parameter modulation.

Our paper is organized as follows. In Section 2, we describe a model of a chaotic communication system with noisy transmitter-receiver channel, and consider several filtration algorithms. Section 3 describes the experimental study and presents a comparison of noise reduction methods. The conclusion and a discussion are presented in Section 4.

\section{Materials and Methods}

\subsection{Synchronization of Rössler Chaotic Systems}

After the discovery of the chaotic synchronization phenomenon, several chaos-based communication techniques were invented: chaotic signal masking [12], chaotic shift keying [13], nonlinear mixing of informational signal into chaotic carrier [14], and chaotic parameter modulation [15] among others. All these types of chaotic communication techniques suffer from noise in the transmitting channel, making them hardly applicable in practice [5].

Let us consider a chaotic communication system with parameter modulation as presented in Figure 1. A digital informational signal $m(t)$ affects the chaotic generator parameter set $P=[a, b, c \ldots]$, forcing it to take value $P_{1}$ (logical 0) or $P_{2}$ (logical 1). In a particular case, only one parameter $p$ can be changed. Slave's parameter has a pre-defined value $P_{1}$. When parameter values are similar, the synchronization error is small; in ideal cases, it converges to zero. Otherwise, the synchronization error is notable; a detector recognizes the input value and matches it with the corresponding logical values 0 or 1 .

The situation significantly changes when noise appears in the signal path. The synchronization error becomes notable in both cases, so the detector needs to be very sophisticated to distinguish logical 0 from 1. At the SNR ratio $\theta>1$, energies of both signals corresponding to 0 and 1 become close, and communication fails.

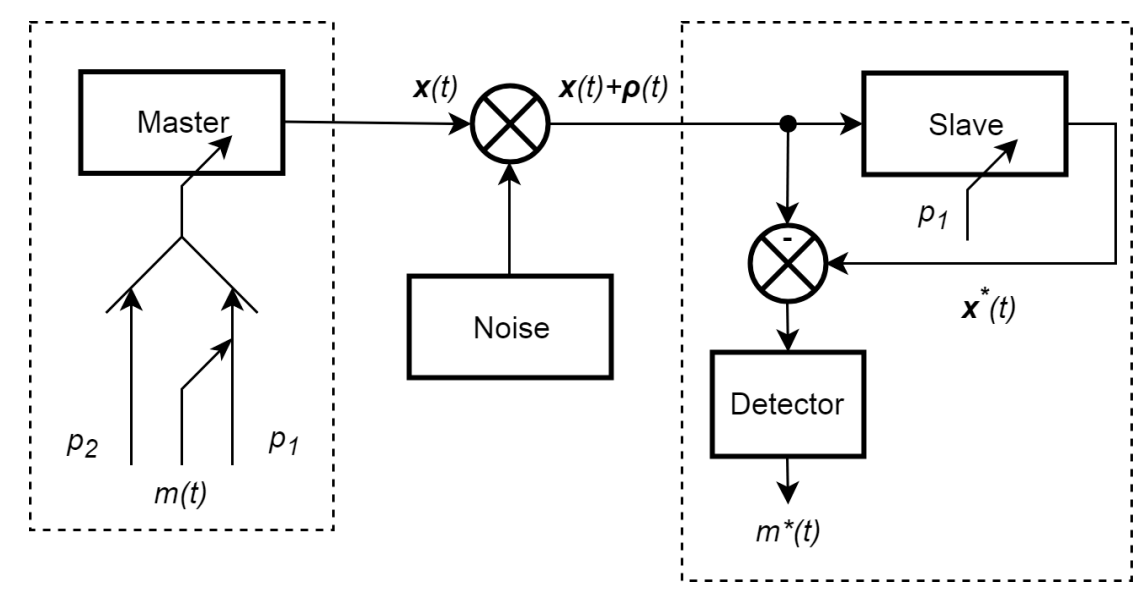

Figure 1. Block diagram of communication system based on Rössler oscillator with parameters modulation.

Let us take Rössler chaotic system, a well-known chaotic oscillator [16]: 


$$
\left\{\begin{array}{l}
\dot{x}=-(y+z) \\
\dot{y}=x+0.2 y \\
\dot{z}=0.2+z(x-5.7)
\end{array}\right.
$$

Synchronization of two chaotic systems in the master-slave topology may be performed using different methods such as Pecora-Carroll technique [17], Hamiltonian forms [18], or the Open-Plus-Close-Loop approach (OPCL) [19]. For our study, we chose the simplest technique of linear feedback control method. The main idea is to include the driving state variable into the driven system with a coefficient $k \in(0 ; 1]$. Thus, we derive the following slave system from the Equation (1):

$$
\left\{\begin{array}{l}
\dot{x}_{s}=-\left(y_{s}+z_{s}\right)+k\left(x_{m}-x_{s}\right) \\
\dot{y}_{s}=x_{s}+0.2 y_{s} \\
\dot{z}_{s}=0.2+z_{s}\left(x_{s}-5.7\right)
\end{array},\right.
$$

here index $s$ stands for driven state variables, index $m$ is the driving ("master") variable, and $k$ is the coupling constant. Value $k=1$ is usually taken. This type of synchronization is available for many known chaotic oscillators, such as the Chua circuit, Sprott simple chaotic flows, and others.

In our case, the Rössler-driven oscillator has 3 parameters: $a=0.2, b=0.2, c=5.7$. We can make logical 1 more or less distinguishable by choosing another parameter set for the master oscillator. To obtain the result presented in Figure 2 we suppose $a=0.2, b=0.2, c=3.5$. Message 0101 was sent in this example. One can see the synchronization error corresponding to this message in Figure $3 a$. The conversion of this signal into binary code can be performed using e.g. envelope detection [20].

Let us consider additive disturbances simulated with a white Gaussian noise source. The same synchronization error in a noisy channel with $\mathrm{SNR}=5 \mathrm{~dB}$ is presented in Figure $3 \mathrm{~b}$. Figure 4 presents the conversion of error signals into digital form using the algorithm described below.

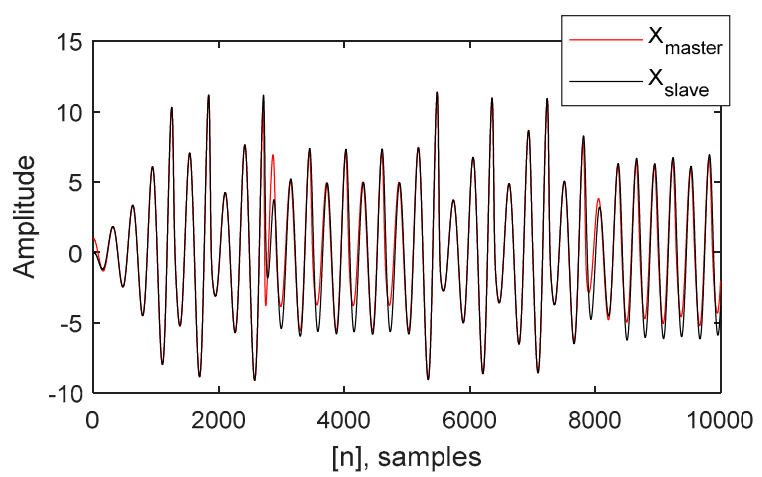

(a)

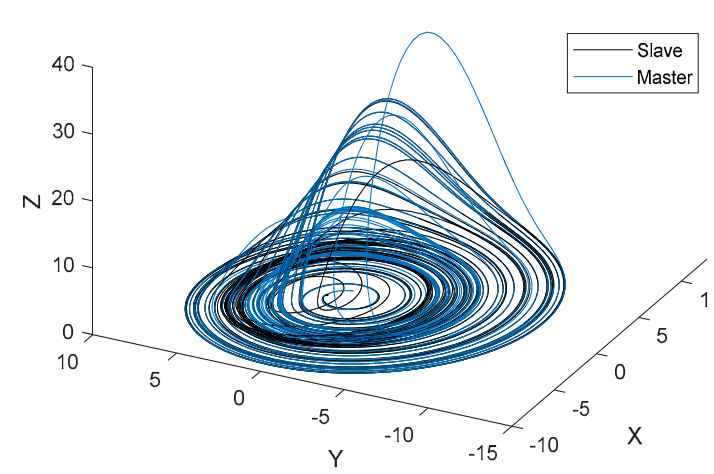

(b)

Figure 2. The dynamics of master (1) and slave (2) systems (a) and corresponding attractors (b).

Notice that in our study, the communication system is only a computer model, so absolute values of variables have no direct physical meaning. The step size and numerical method for chaotic oscillators' simulation were taken according to [21,22].

The algorithm of detection block is schematically presented in Figure 5. It consists of a peak detector and threshold block. This design was developed as a sample detector, showing the fundamental possibility of the chaotic communication system to work. In real systems, more advanced detectors can be used, including envelope fitters and other sophisticated components.

Figure 6 illustrates the dependence between quality of synchronization and the values of parameter $c$ and SNR. One can observe the tendency of synchronization quality enhancement while the value of $c$ is ascending. 


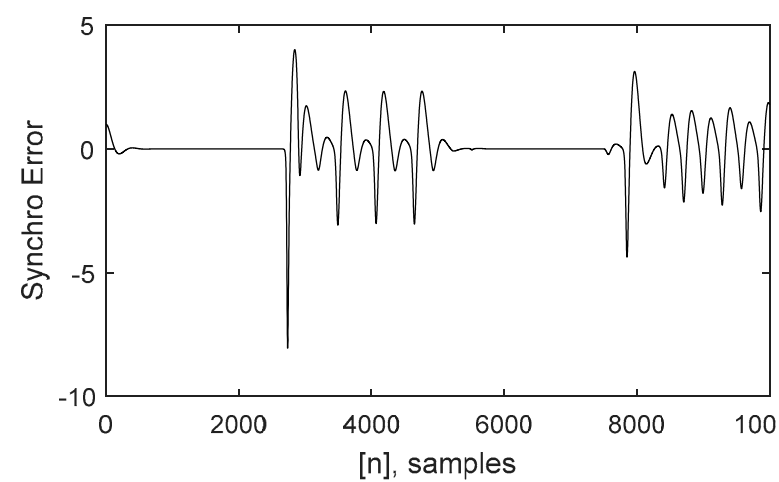

(a)

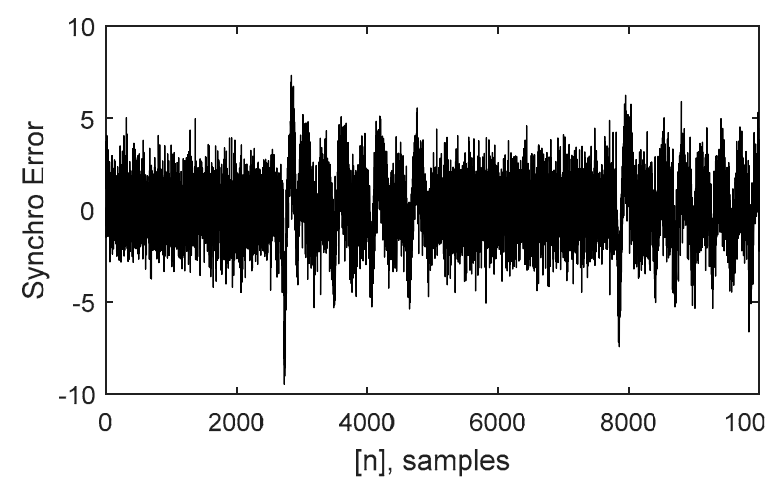

(b)

Figure 3. Master-slave synchronization error with ideal (a) and noisy (b) synchronization signal.

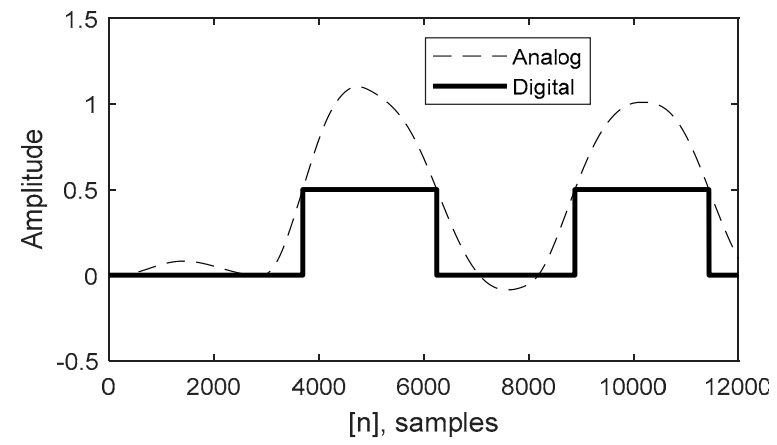

(a)

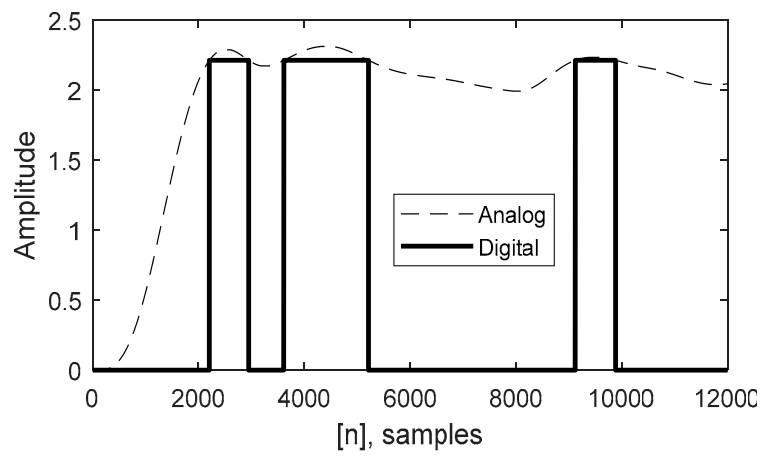

(b)

Figure 4. Correct message recognition in ideal channel (a) and incorrect recognition in noised channel $(\mathrm{SNR}=5 \mathrm{~dB})(\mathbf{b})$.

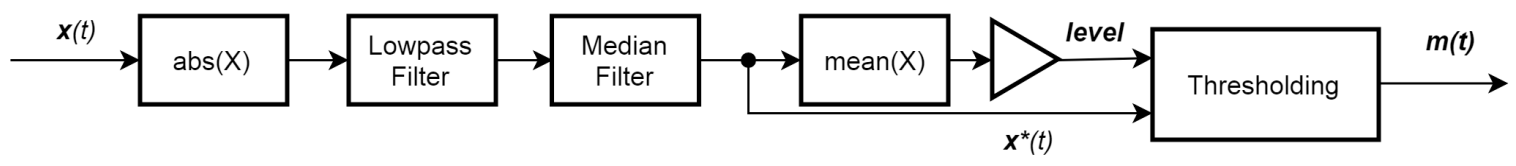

Figure 5. The scheme of detection block.

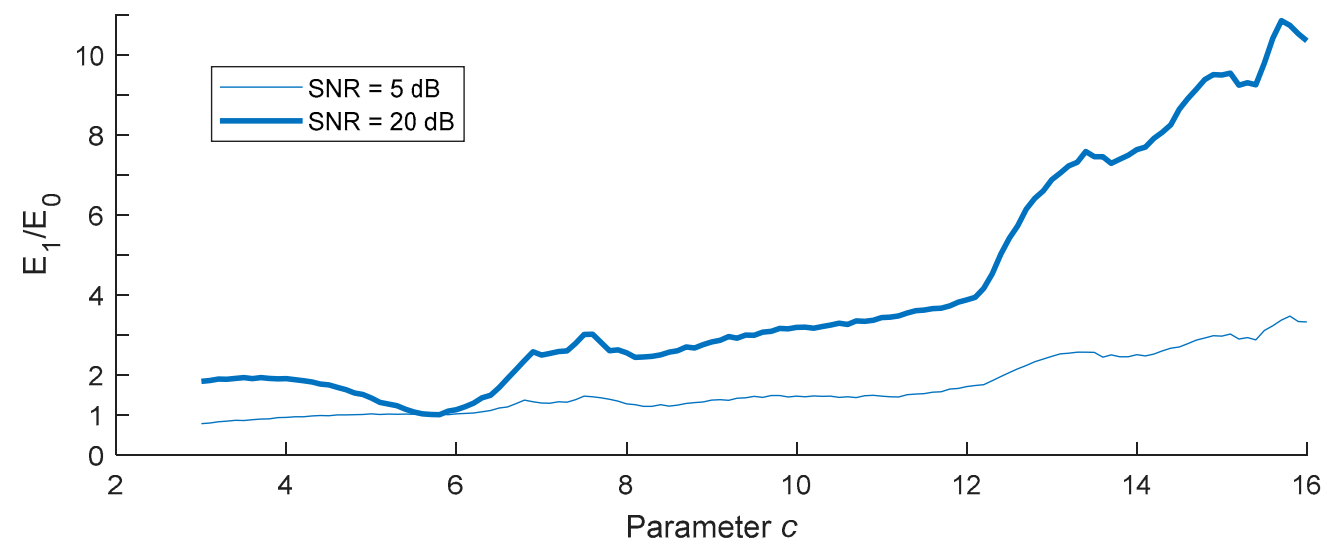

Figure 6. RMS ratios for synchronization error in case of logical $1\left(E_{1}\right)$ and logical $0\left(E_{0}\right)$ transmission depending on parameter $c$ value. Levels equal and lower than $E_{1} / E_{0}=1$ corresponds to the case of total message loss. 
Nevertheless, even for the higher values of parameter $c$, low SNR still means low difference between logical 0 and 1 . This may result in high levels of corruption of the received messages. For many real applications, SNR $\leq 5 \mathrm{~dB}$ is common value [20], so some kind of filtration of the noisy message signal could be performed to improve the SNR ratio. The experiments described below were performed with $c=3.5$ to make the use of filtering techniques more indicative.

\subsection{Filtration Techniques}

Numerous filtration techniques may be applied to denoise communication signals: recursive and non-recursive filtering [23], median filtering [23], algorithms based on discrete wavelet transform [24], Wiener filters [25], and empirical mode decomposition (EMD) [26,27]. The filter is placed at the first stage of the receiver, as presented in Figure 7.

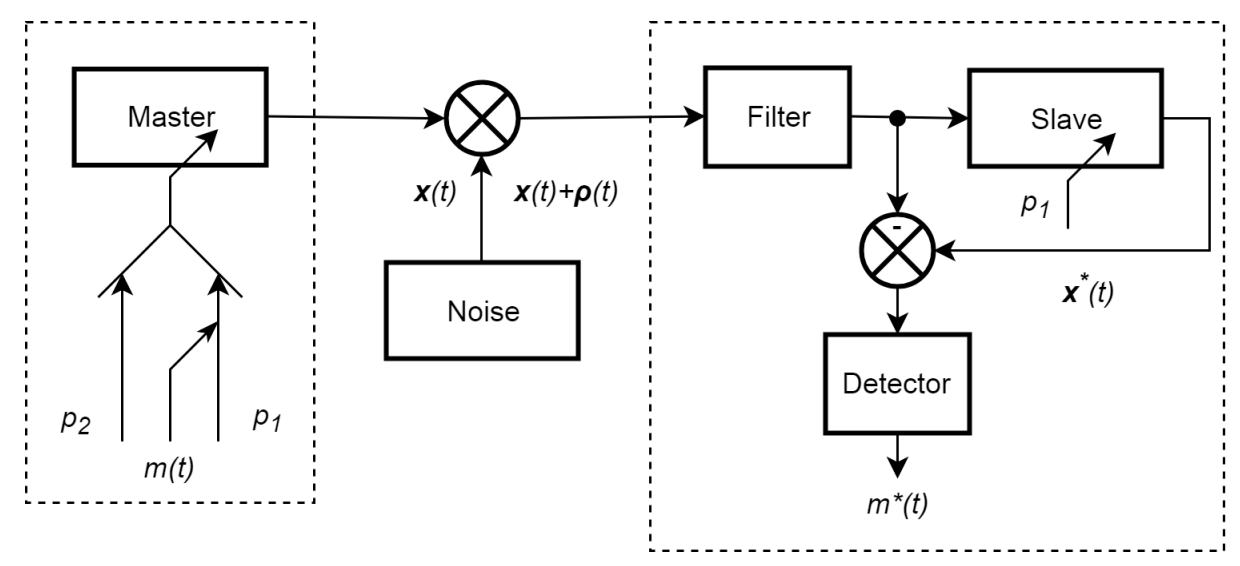

Figure 7. Chaotic communication system with filter.

A description of abovementioned methods can be found in referenced publications [23-27]. In this paper, we focus on two filtration techniques: EMD, as a relatively little known technique whose usage in chaotic communications has never been reported; and a novel approach involving chain of driven oscillators.

\subsubsection{Empirical Mode Decomposition}

EMD (empirical mode decomposition) is a signal decomposition method alternative to Fourier transform and wavelet transform [28]. The basic functions of EMD are unknown a priori, and can be found computationally during the decomposition process. Thus, EMD makes it possible to decompose a signal into non-stationary and nonlinear intrinsic mode functions (IMF). Discarding noise-specific modes in the resulting decomposition, one can restore original signal, keeping it non-stationary and nonlinear. The last property becomes critical when processing chaotic signals, which are nonlinear by their nature, and non-stationary (considering modulation in communication tasks).

Empirical mode (EM) or internal oscillation (IMF) is a function that has the following two quantitative properties:

1. The number of extrema (maxima and minima) and the number of zero crossings should not differ by more than one:

$$
N_{\max }+N_{\min }=N_{\text {zero }} \pm 1,
$$

where $N_{\max }$ is the quantity of maxima; $N_{\min }$ is the quantity of minima; and $N_{z e r o}$ is the quantity of zero-crossings. 
2. The average value determined by two envelopes (upper and lower) must be equal to zero. When processing real-world signals, it should not exceed some small value determined by computer accuracy and errors associated with the data acquisition, transformation and transmission.

$$
\frac{U(k)+L(k)}{2} \leq \varepsilon, k \in \overline{1 \ldots N},
$$

where $U(k)$ is the upper envelope; $L(k)$ is the lower envelope; and $N$ is the number of signal samples.

These empirical mode properties make it possible to apply Hilbert spectral analysis to them.

The EMD algorithm represents the given discrete signal as a set of EMs [29]. The algorithm is based on the fundamental idea that at each stage, the signal (current residue) is represented as a sum of the rapidly oscillating components (extracted EM), and slowly oscillating ones (residue). The latter is further decomposed if it has at least one minimum and one maximum.

The algorithm can be written as the following sequence of steps:

Step 1. Consider the current residue $r_{p}(k)\left(r_{1}(k)\right.$ - the first residue equal to the source signal $\left.s(k)\right)$. Its extrema are determined and split into sets: $\left\{M_{i}\right\}, i=1,2,3, \ldots K, \ldots$ and $\left\{m_{i}\right\}, i=1,2,3, \ldots K, \ldots$ - set of maxima and minima, respectively.

Step 2. Two envelopes are built with the cubic splines interpolation fitting the found extrema: $U_{j}(k)=f_{U}\left(M_{i}, k\right), L_{j}(k)=f_{L}\left(m_{i}, k\right)$ - upper and lower envelopes, respectively, $j$ is the iteration of the sifting process (see below).

Step 3. After building the envelopes, their half-sum is calculated; the local (instantaneous) average value depending on time:

$$
e_{j}(k)=\frac{U_{j}(k)+L_{j}(k)}{2} .
$$

Step 4. The average value is subtracted from the current residue and the obtained result $h_{j}(k)$ becomes a "candidate" to be the next mode:

$$
\begin{gathered}
h_{j}(k)=r_{p}(k) ; j=1 \\
h_{j}(k)=r_{p}(k)-e_{j}(k) ; j>1
\end{gathered}
$$

where $p$ is the number of the extracted mode.

In this step, it is necessary to check conditions (3) and (4) to determine if the empirical function $h(k)$ can be classified as empirical mode or not. If both conditions are met, then proceed to step 5 . Otherwise, the algorithm returns to step 1 with $h_{j}(k)$ as the current residue. Such an iterative procedure is called a "sifting process", which can be written as follows:

$$
\begin{gathered}
h_{1}(k)=r_{p}(k) ; h_{2}(k)=h_{1}(k)-e_{1}(k) \\
h_{j+1}(k)=h_{j}(k)-e_{j}(k) ; c_{p}(k)=h_{i t e r}(k)
\end{gathered}
$$

here, $e_{j}(k)$ is the average value of the function on the $j$-th iteration of the sifting process; $h_{j}(k)$ is the current result of the $j$-th iteration of the sifting process; and iter is the total number of iterations for the given EM.

At the iteration with the iter number, the sifting process for the next mode extraction is stopped and the transition to step 5 is performed.

Step 5. After the EM is extracted in its final form, it is subtracted from the current residue to form a new residue (to update the residue): $r_{p+1}(k)=r_{p}(k)-c_{p}(k) ; c_{p}(k)$ is the obtained EM; $r_{p}(k)$ is the current residue, and $r_{p+1}(k)$ is the new residue.

Step 6. Next, $r_{p+1}(k)$ is used as a residue to perform the algorithm form step 1 .

Figure 8 shows the diagram of the algorithm. 


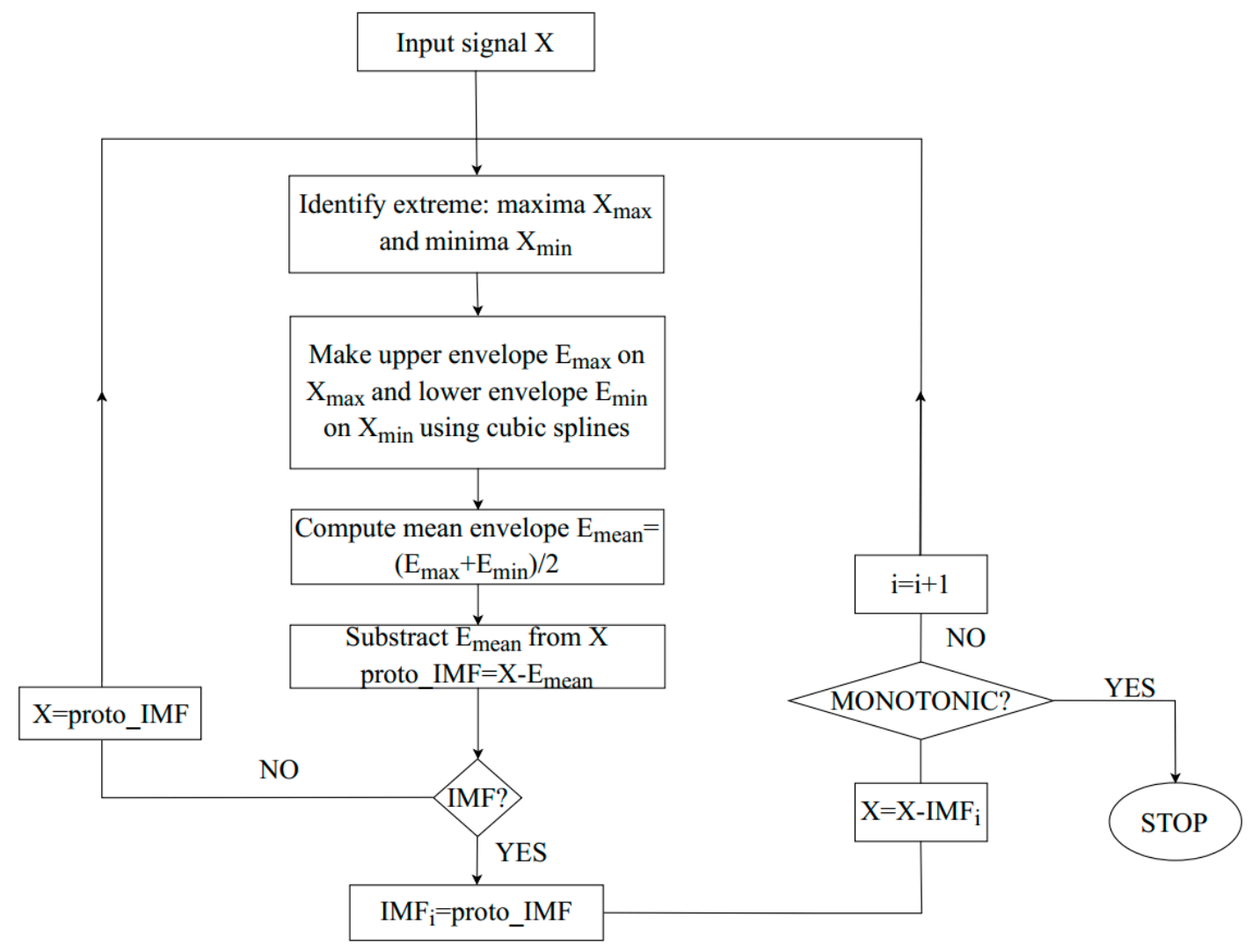

Figure 8. The EMD algorithm.

The algorithm has an empirical nature. Currently, it has no deeply-studied theoretical basis compared to classical Fourier analysis or wavelet analysis.

Based on practical results, an empirical formula was derived to estimate the total number of EMs in the decomposition of an arbitrary signal [29]:

$$
M=\log _{2} N \pm 1,
$$

here, $M$ is the total EM quantity, and $N$ is number of samples in the signal.

Using EMD, the original signal can be restored completely:

$$
s(k)=\sum_{p=1}^{M} c_{p}(k)+r(k)
$$

or partly:

$$
s(k)=\sum_{p \in P} c_{p}(k)+r(k),
$$

where $P$ is the set of selected EMs.

Considering EMD of a noisy signal, one can easily understand that EMs with small numbers correspond to noise, and with higher numbers correspond to a useful signal. All noise reduction algorithms utilizing EMD are based on this idea. A number of such algorithms have been developed, e.g., ensemble EMD [26], conventional EMD, and iterative EMD [27]. As an example, we used so-called conventional EMD (CEMD). This algorithm is based on the assumption that the energy of "pure noise" EMs decreases according to the law:

$$
\hat{E}_{p}=\frac{\hat{E}_{1}}{0.719} 2.01^{-p}, p=2,3,4 \ldots,
$$


here, $p$ is the number of EM; and $\hat{E}_{p}$ is the energy of EM.

The cleaned signal can be written in the following form:

$$
\widetilde{x}(t)=\left.\sum_{p=1}^{M} c_{p}\right|_{E_{p}>\hat{E}_{p}}(t)+r(t),
$$

i.e. only those of EM are summarized, the energy of which exceeds the energy of the "pure noise" EM due to the presence of the useful signal.

\subsubsection{Nonlinear Filtration by Chain of Driven Oscillators}

It is known that chaotic motion can be observed in state variable filters [30]. Thus, the reverse statement that chaotic systems can work as filters can also be valid. In our study, we propose a non-linear filter construction as a cascade of driven oscillators. The number of oscillators in the chain defines the order of such a filter. Figure 9 presents a chain involving four oscillators. Notice that the first driven oscillator works as a filter from Figure 7, the second oscillator works as a signal amplifier to feed the next oscillator, and so on. Primary experiments made with a four-oscillator filter have shown promising performance.

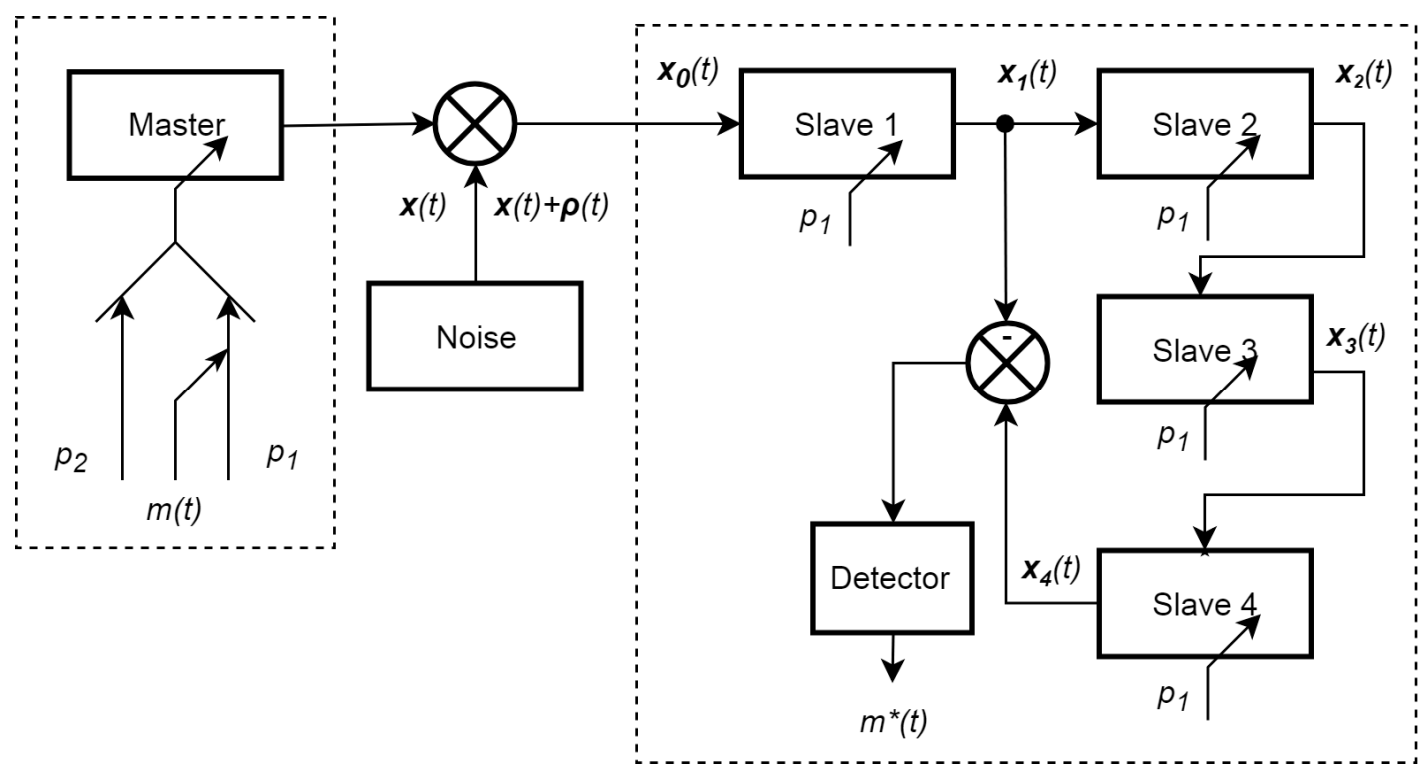

Figure 9. The chain of driven oscillators involving 4 oscillators.

Analysis of signal propagating from point $x_{0}$ to $x_{4}$ shows the process of information extraction and amplification, see Figure 10.

Notice that noise superimposed on signal of logical 0 also tends to be amplified, so a more sophisticated filter could be designed which was capable of analyzing the signal not only at point $x_{4}$, but also at other points. 


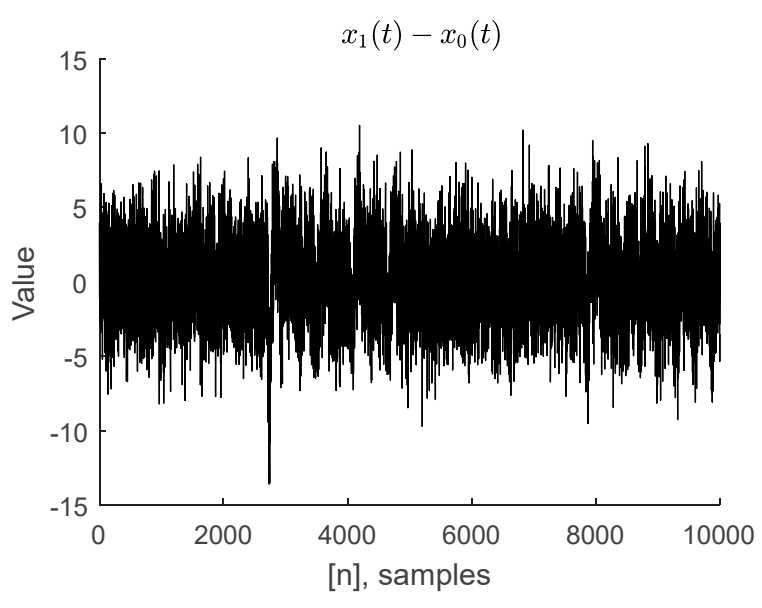

(a)

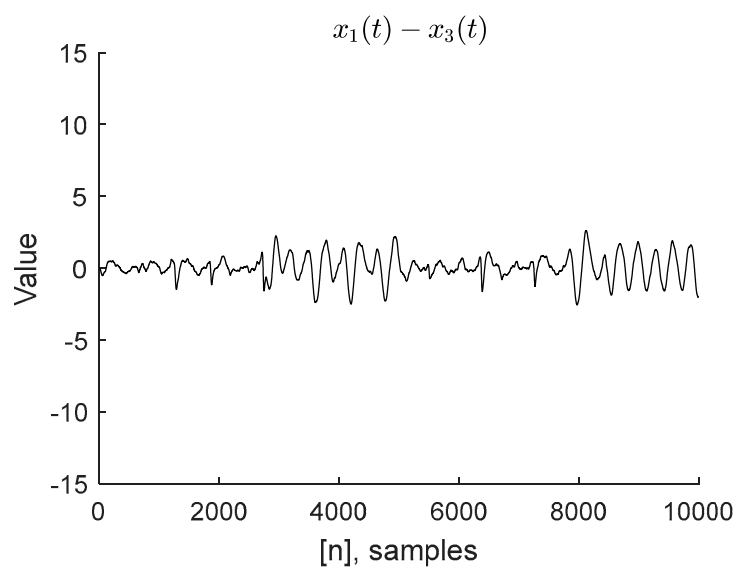

(c)

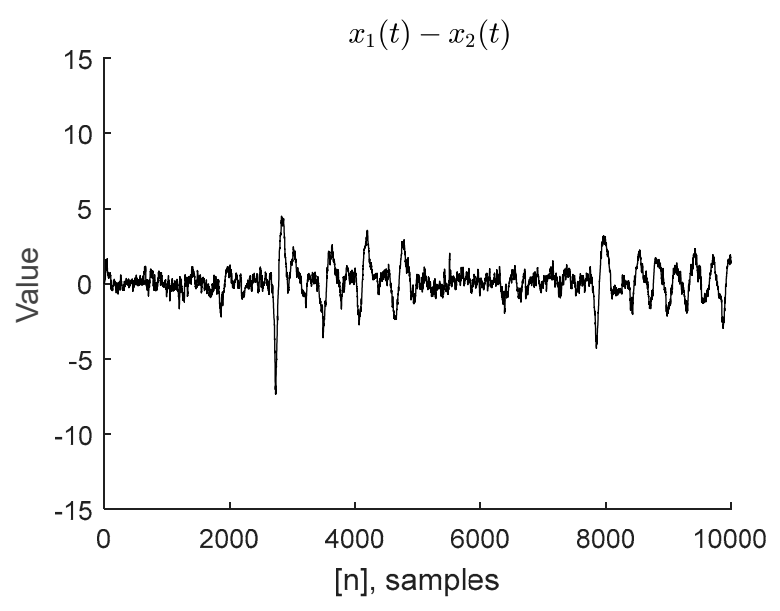

(b)

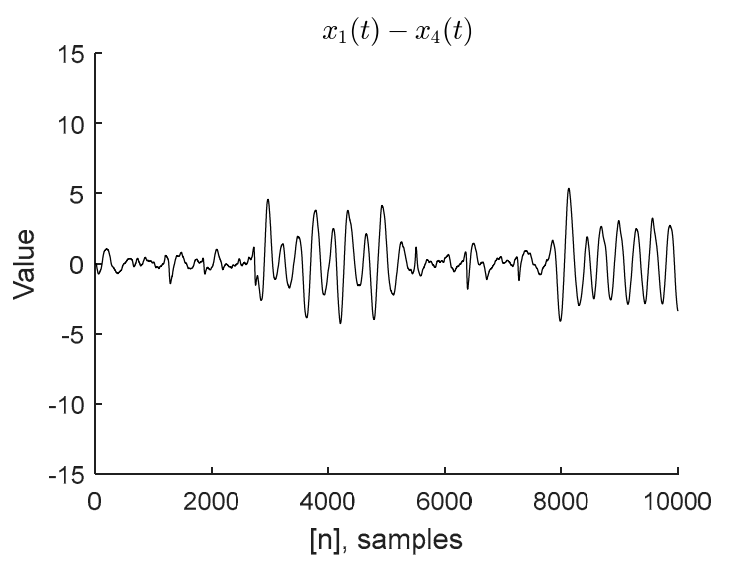

(d)

Figure 10. Restored message in noisy sequence $0101(\mathrm{SNR}=0 \mathrm{~dB})$ at the first stage $(\mathbf{a})$, second stage $(\mathbf{b})$, third stage (c) and forth stage (d).

\section{Results}

For a relative comparison of filtration techniques, we used the following approach. First, all filtering techniques were implemented as MATLAB functions. Different messages were passed though the model of chaotic communication system based on Rössler oscillators, as described in Section 2. Filtered synchronization signals were sent to the driven oscillator and its responses were restored as digital messages using the same detector (Figure 5). After a number of experiments with the same SNR value (100 or 200), we increased the intensity of noise source. The whole experiment was made for SNR values from -30 to $20 \mathrm{~dB}$. For $\mathrm{SNR}=20 \mathrm{~dB}$, no filtration was required for stable messages recognition. For $\mathrm{SNR}=-30 \mathrm{~dB}$, no algorithm was able to restore any message.

Experimental results are generalized in Figure 11. One can see that nonlinear filtration using the chain of driven oscillators shows the best performance, slightly better than wavelet technique accepted by different researches as suitable for chaotic signals denoising [4,9]. The EMD filtration technique shows best performance for low SNR values. IIR filtering and median filtering also enhance the correct bits rate, but not so efficiently.

It should be taken into account that these results were obtained on an ideal system with pure white noise affecting messages in channel. Notwithstanding, we got a confirmation of the validity of our approach involving chain of driven oscillators. It appears to be more efficient than EMD, wavelet and median filtration. EMD also shows good performance, making it practically applicable. 


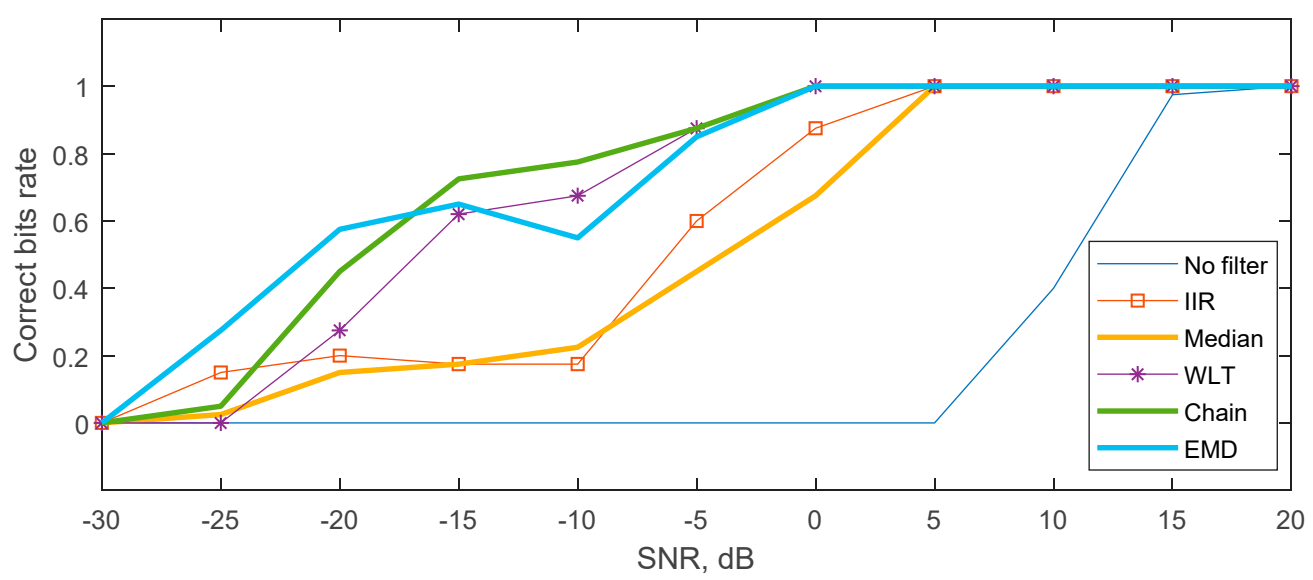

Figure 11. Ratio of correctly recognized bits according to SNR level in communication channel and filtration method: IIR—4-th order low-pass Butterworth filter; MED—-median filter; WLT—wavelet transform filter using external phase Daubechies wavelet with 8 vanishing moments; EMDconventional EMD filter; Chain- chain of driven oscillators technique.

The first example is presented in Figure 12. The black waveform represents the master-slave synchronization error when transferring the message 01010101 without a noise in channel (compare to Figure 3a), blue dotted waveform represents the synchronization error with the filtered noisy $(\mathrm{SNR}=-10 \mathrm{~dB})$ synchronization signal. Original synchronization error waveform (Figure 12a) may be seen to be unlike the signal in Figure 12b.

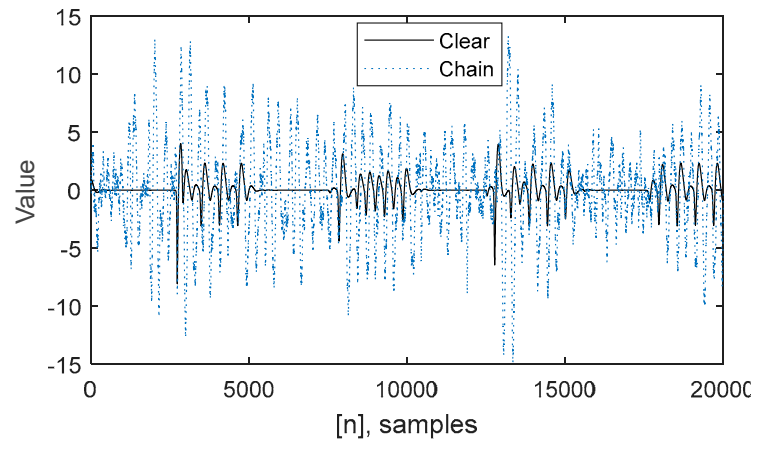

(a)

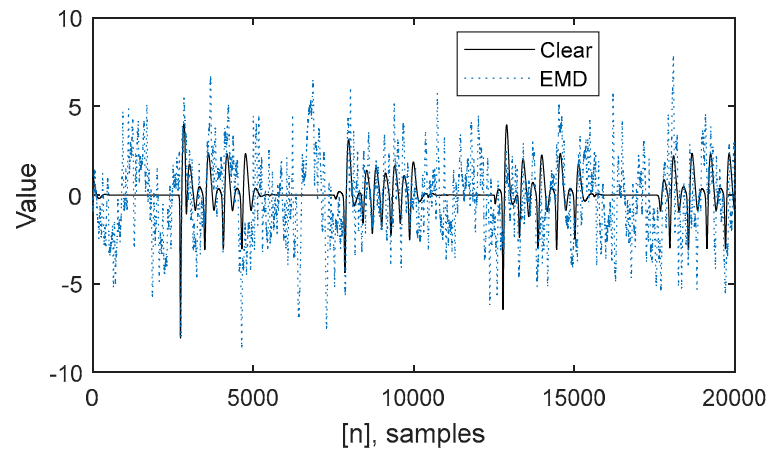

(b)

Figure 12. Recovering of master-slave synchronization error at $\mathrm{SNR}=-10 \mathrm{~dB}$ using chain of driven oscillators denoising (a) and EMD filtration (b).

Figure 13 reveals the details of the experiment. The same message 01010101 passes through the chaotic communication channel. The upper waveform is the master-slave synchronization error without noise in the channel (same as in Figure 3a), and a square envelope digital message encoded in synchronization error signal. Other analog waveforms were obtained from the detector. In this example, only the oscillators' chain method allows us to recognize the original message. After EMD, the 3rd and 4th bits are missing, the median filter has lost the second and fourth logical 1, and other methods are total loss. This example also illustrates the imperfection of the detector used, but demonstrates the superiority of the chain of driven oscillators approach well. 


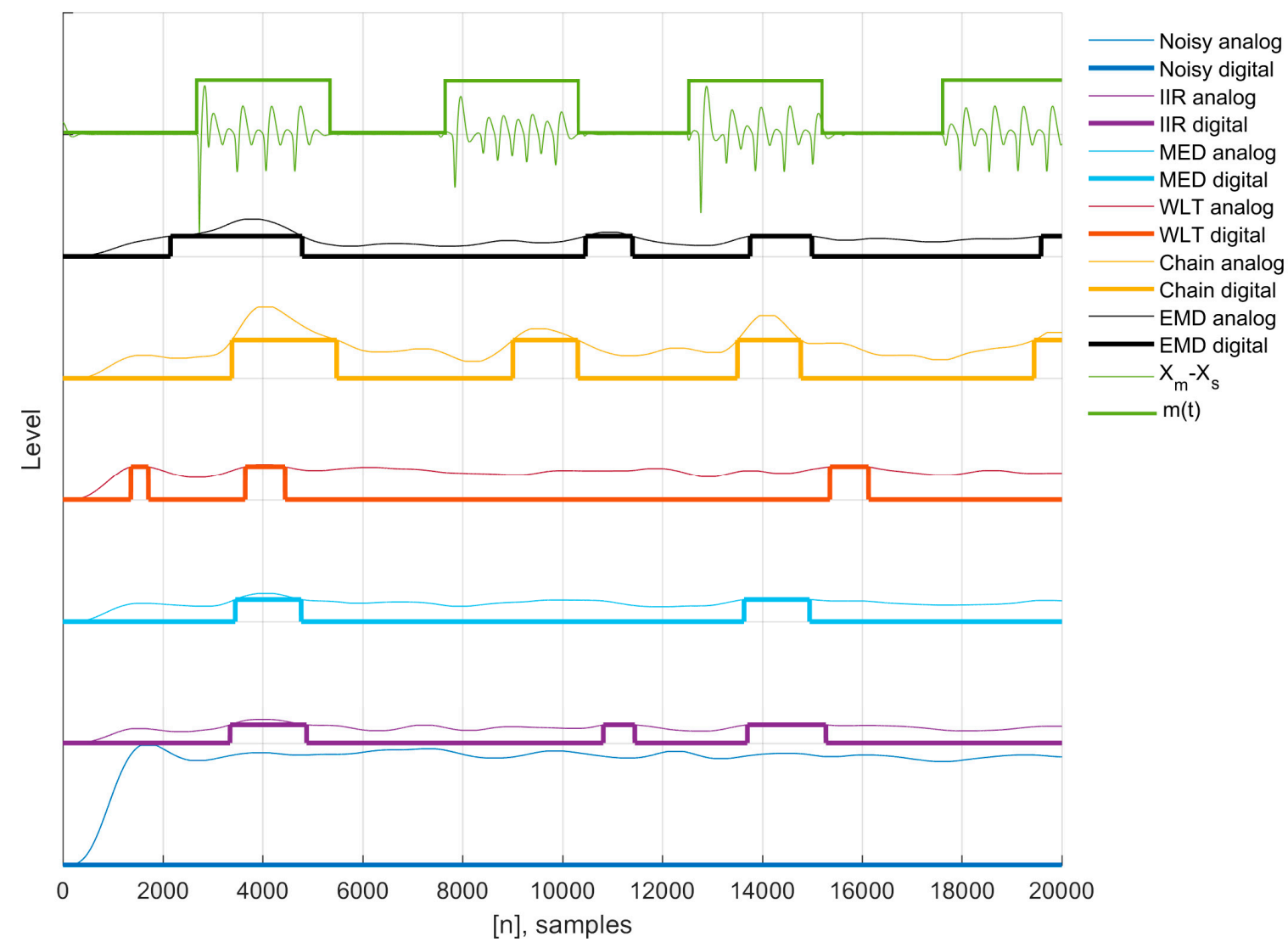

Figure 13. Example of a message recognition, $\mathrm{SNR}=-10 \mathrm{~dB}$. Noisy analog indicates without filtering; IIR indicates 4-th order low-pass Butterworth filter; MED indicates median filter; WLT indicates maximal overlap discrete wavelet transform filter using external phase Daubechies wavelet with 8 vanishing moments; EMD indicates conventional EMD filter; Chain indicates 4 driven oscillators chain filter; $X_{m}-X_{s}$ indicates master-slave synchronization error with no noise in channel; $m(t)$ indicates original digital message waveform.

To make an important step towards practical implementation of the chaotic communicational system, we should take into account that the relative performance of filtration methods presented in Figure 11 is independent to the disturbance type. Often, disturbance is modeled by noise, including colored noise [31], though other signals also may be considered as disturbances [32]. Noise classification involves conception of the noise color, i.e. dependency of noise power from frequency. Colored noise has power spectral density of the form $S(f) \propto 1 / f^{\alpha}$, where $\alpha \in[-2 ; 2]$. White noise has flat spectrum $(\alpha=0)$, while the spectral density of a pink noise compared to white noise is attenuated by 3 decibels per octave $(\alpha=1)$, and the spectral density of a blue noise increases by 3 decibels per octave $(\alpha=-1)$. White noise is an equivalent of uniformly-disturbed media; pink noise can be naturally generated by great amount of physical processes; and blue noise may be a good analog of radio waves propagating media where higher frequencies are occupied by more powerful sources. Real world ambient noises can often be approximated with fractional $\alpha$ values [31].

Figure 14 shows a comparison of studied filtering techniques in the presence of colored noises. The problem with pink noise is that chaotic systems have spectra of the same type, i.e., decaying in higher frequencies, so the presence of pink noise masks the chaotic signal and, consequently, weakens the performance of the filtration by the chain of chaotic oscillators. Instead, blue noise can be called 'chaos friendly', but EMD needs to be properly adjusted when higher harmonics appear. In general, this experiment confirms the abovementioned results of relative performance of the filtration techniques. 


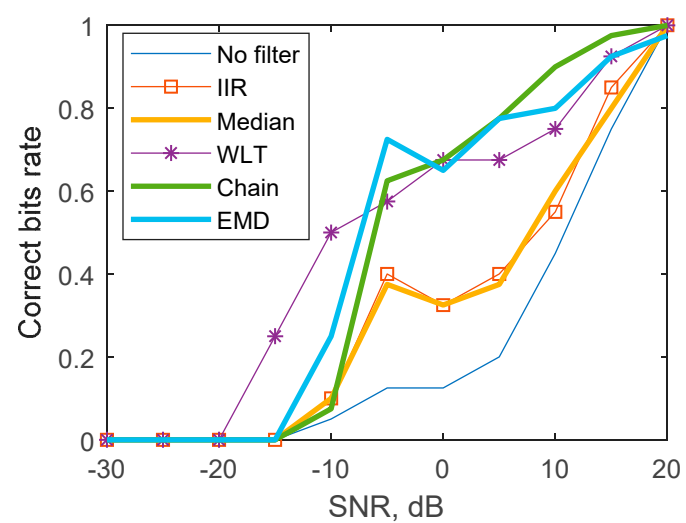

(a)

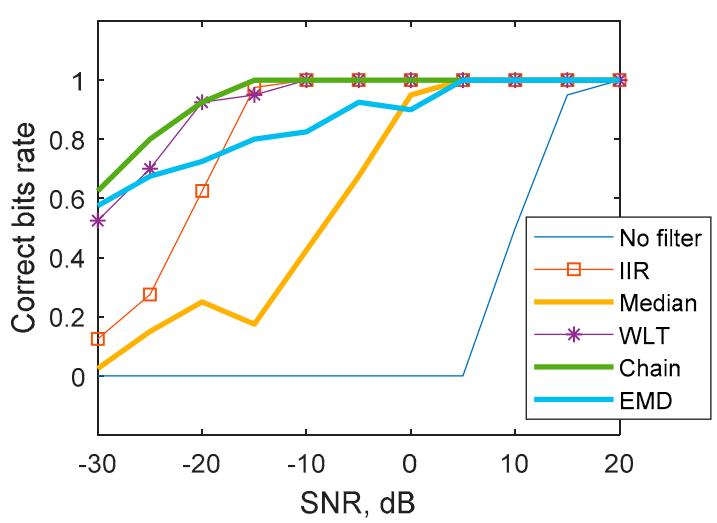

(b)

Figure 14. Ratio of correctly recognized bits according to SNR level in communication channel, noise color indicates pink (a) and blue (b), and filtration method: IIR indicates 4-th order low-pass Butterworth filter; MED indicates median filter; WLT indicates a wavelet transform filter using external phase Daubechies wavelet with 8 vanishing moments; EMD indicates the conventional EMD filter; Chain indicates the chain of driven oscillators technique.

Table 1 summarizes studied denoising methods applied for chaotic communications.

Table 1. Brief comparison of nonlinear denoising techniques for chaotic signals.

\begin{tabular}{ccc}
\hline Denoising technique & Pros & Cons \\
\hline Median filtering & Easy to implement; & Not efficient \\
one adjustable parameter & Fine performance & Difficult to adjust \\
EMD denoising & Fine performance & Difficult to adjust; \\
& Fine performance; & \\
Driven oscillators chain filtering & easy to implement; & \\
& no parameters to adjust & \\
\hline
\end{tabular}

\section{Conclusion and Discussion}

Following previous research, we demonstrate the possibility of chaotic communication signals to be effectively denoised when messages are affected by disturbances in a communication channel. As for conventional communication systems, the use of proper denoising algorithms significantly enhances the noise resistivity of communication. Denoising techniques can determine the operation ability of the investigated system in a case of low signal-to-noise ratio. Using white noise as a disturbance, we obtained the correct bit-rate approximately $50 \%$ when signal-to-noise ratio was $-20 \mathrm{~dB}$ using our novel nonlinear filtration method based on chain of driven oscillators. This method and empirical mode decomposition denoising were more effective and easier to adjust than the wavelet technique used for denoising in chaotic communications by other researchers.

Moving towards practical system implementation, we studied the expected behavior of the system in presence of different noise types. Numerical experiments with pink and blue noises show that the performance of the whole communication system is influenced by noise type, but relative denoising methods effectiveness is not. Also, it was found that basic chaotic oscillator parameters variation may result in notable improvement of noise resistance.

The obtained results can be applied to other types of chaos-based communication schemes based on chaotic synchronization principle.

Our further investigations will include enhancements of the detection algorithm, improving the filtration algorithm based on EMD, and comprehensive study of the nonlinear filtering method 
based on the slave generators cascading. In particular, we will try to determine optimal cascade number and develop the theory of cascaded chaotic filters. We will also implement the chaotic communication system utilizing state-of-art message encoding and perform some experiments with hardware prototypes, including hydroacoustic and underwater communications systems.

Author Contributions: Conceptualization, D.B. and D.K.; Data curation, T.K. and V.A.; Formal analysis, D.B. and A.V.; Investigation, T.K., A.V., V.A. and V.O.; Methodology, D.B. and A.V.; Project administration, D.B. and D.K.; Resources, T.K. and D.K.; Software, T.K., A.V., D.K. and V.A.; Supervision, D.B. and D.K.; Validation, D.B., T.K., V.A. and V.O.; Visualization, V.A.; Writing-original draft, D.B., T.K. and V.O.; Writing-review \& editing, D.K. and V.O.

Funding: This research was funded by Russian Science Foundation (Project NO 17-71-20077).

Acknowledgments: Special thanks to our young colleagues from CAD department of Saint-Petersburg Electrotechnical University "LETI" for their participation.

Conflicts of Interest: The authors declare no conflict of interest.

\section{References}

1. Dmitriev, A.S.; Hasler, M.; Panas, A.I.; Zakharchenko, K.V. Basic principles of direct chaotic communications. In Synchronization: Theory and Application; Springer: Dordrecht, The Netherlands, 2003; pp. 41-63.

2. Riaz, A.; Ali, M. Chaotic Communications, their applications and advantages over traditional methods of communication. In Proceedings of the 6th International Symposium on Communication Systems, Networks and Digital Signal Processing, Graz, Austria, 25 July 2008; pp. 21-24. [CrossRef]

3. Prokhorov, M.D.; Ponomarenko, V.I.; Kulminskiy, D.D.; Koronovskii, A.A.; Moskalenko, O.I.; Hramov, A.E. Resistant to noise chaotic communication scheme exploiting the regime of generalized synchronization. Nonlinear Dyn. 2017, 87, 2039-2050. [CrossRef]

4. Kaddoum, G. Wireless Chaos-Based Communication Systems: A Comprehensive Survey. IEEE Access 2016, 4, 2621-2648. [CrossRef]

5. Koronovskii, A.A.; Moskalenko, O.I.; Hramov, A.E. On the use of chaotic synchronization for secure communication. Uspekhi Fizicheskikh Nauk 2009, 179, 1281-1310. [CrossRef]

6. Liu, L.; Li, Y.; Zhang, Z.; Song, H.; Jin, Z. High-efficiency and noise-robust DCSK approach based on an analytically solvable chaotic oscillator. Electron. Lett. 2018, 54, 1384-1385. [CrossRef]

7. Williams, C. Robust chaotic communications exploiting waveform diversity. Part 1: Correlation detection and implicit coding. IET Commun. 2008, 2, 1213-1222. [CrossRef]

8. Jako, Z.; Kis, G. Application of noise reduction to chaotic communications: A case study. IEEE Trans. Circ. Syst. I Fundam. Theory Appl. 2000, 47, 1720-1725. [CrossRef]

9. An, X.L.; Yu, J.N.; Li, Y.Z.; Chu, Y.D.; Zhang, J.G.; Li, X.F. Design of a new multistage chaos synchronized system for secure communications and study on noise perturbation. Math. Comput. Model. 2011, 54, 7-18. [CrossRef]

10. Hasler, M.; Schimming, T. Chaos communication over noisy channels. Int. J. Bifurc. Chaos 2000, 10, 719-735. [CrossRef]

11. Wang, S.; Long, Z.; Wang, J.; Guo, J. A noise reduction method for discrete chaotic signals and its application in communication. In Proceedings of the 4th International Congress on Image and Signal Processing, Shanghai, China, 15-17 October 2011; pp. 2303-2307. [CrossRef]

12. Cuomo, K.M.; Oppenheim, A.V.; Strogatz, S.H. Synchronization of Lorenz-based chaotic circuits with applications to communications. IEEE Trans. Circ. Syst. II Analog Digit. Signal Process. 1993, 40, 626-633. [CrossRef]

13. Dedieu, H.; Kennedy, M.; Hasler, M. Chaos shift keying: Modulation and demodulation of a chaotic carrier using self-synchronizing Chua's circuits. IEEE Trans. Circ. Syst. II Analog Digit. Signal Process. 1993, 40, 634-642. [CrossRef]

14. Dmitriev, A.S.; Panas, A.I.; Starkov, S.O. Experiments on speech and music signals transmission using chaos. Int. J. Bifurc. Chaos 1995, 5, 1249-1254. [CrossRef]

15. Yang, T.; Chua, L. Secure communication via chaotic parameter modulation. IEEE Trans. Circ. Syst. I Fundam. Theory Appl. 1996, 43, 817-819. [CrossRef]

16. Rössler, O.E. An equation for continuous chaos. Phys. Lett. A 1976, 57, 397-398. [CrossRef] 
17. Pecora, L.; Carroll, T. Synchronization in chaotic system. Phys. Rev. Lett. 1990, 64, 821. [CrossRef] [PubMed]

18. Sira-Ramirez, H.; Cruz-Hernandez, C. Synchronization of chaotic systems: A generalized Hamiltonian systems approach. Int. J. Bifurc. Chaos 2001, 11, 1381-1395. [CrossRef]

19. Jackson, E.A.; Grosu, I. An open-plus-closed-loop (OPCL) control of complex dynamic systems. Phys. D Nonlinear Phenom. 1995, 85, 1-9. [CrossRef]

20. Carroll, T.L. Noise-resistant chaotic synchronization. Phys. Rev. E 2011, 64, 015201. [CrossRef] [PubMed]

21. Butusov, D.N.; Ostrovskii, V.Y.; Pesterev, D.O. Numerical analysis of memristor-based circuits with semi-implicit methods. In Proceedings of the 2017 IEEE Russia Section Young Researchers in Electrical and Electronic Engineering Conference, ElConRus, St. Petersburg, Russia, 1-3 February 2017; pp. 271-276.

22. Butusov, D.N.; Andreev, V.S.; Pesterev, D.O. Composition semi-implicit methods for chaotic problems simulation. In Proceedings of the 19th International Conference on Soft Computing and Measurements, SCM, St. Petersburg, Russia, 25-27 May 2016; pp. 107-110.

23. Mitra, S.K. Digital Signal Processing, 2nd ed.; McGraw-Hill: New York, NY, USA, 2001.

24. Percival, D.B.; Walden, A.T. Wavelet Methods for Time Series Analysis; Cambridge University Press: Cambridge, UK, 2000.

25. Brown, R.G.; Hwang, P.Y.C. Introduction to Random Signals and Applied Kalman Filtering, 3rd ed.; John Wiley \& Sons: New York, NY, USA, 1996; ISBN 0-471-12839-2.

26. Taebi, A.; Mansy, H.A. Noise cancellation from vibrocardiographic signals based on the ensemble empirical mode decomposition. J. Biotechnol. Bioeng. 2017, 2, 00024. [CrossRef]

27. Kopsinis, Y.; McLaughlin, S. Empirical Mode Decomposition Based Denoising Techniques; IDCOM, School of Engineering and Electronics the University of Edinburgh, King's Buildings: Edinburgh, Scotland, 2010.

28. Huang, N.E.; Shen, Z.; Long, S.R.; Wu, M.C.; Shih, H.H.; Zheng, Q.; Yen, N.C.; Tung, C.C.; Liu, H.H. The empirical mode decomposition and the Hilbert spectrum for nonlinear and non-stationary time series analysis. Proc. R. Soc. Lond. A 1998, 454, 903-995. [CrossRef]

29. Huang, N.E.; Shen, S.S.P. Hilbert-Huang Transform and Its Applications; World Scientific: London, UK, 2005; ISBN 978-9812563767.

30. Petrzela, J. On the existence of chaos in the electronically adjustable structures of the state variable filters. Int. J. Circ. Theory Appl. 2016, 44, 1779-1797. [CrossRef]

31. Kasdin, N.J.; Walter, T. Discrete simulation of power law noise (for oscillator stability evaluation). In Proceedings of the 1992 IEEE Frequency Control Symposium, Hershey, PA, USA, 27-29 May 1992; pp. 274-283. [CrossRef]

32. Belkin, D.A.; Krasilnikov, A.V.; Pesterev, D.O.; Karimov, T.I. Influence of disturbances in sync signal path on dynamical systems synchronization. In Proceedings of the IEEE Soft Computing and Measurements (SCM), St. Petersburg, Russia, 24-26 May 2017; pp. 145-147. [CrossRef] 